\title{
Plasma and Tissue Lipid Peroxides in Spontaneously Diabetic Chinese Hamsters
}

\author{
Masaaki ETo, ${ }^{1, *}$ Kiyoshi Watanabe, ${ }^{1}$ Yasunori IWASHIMA, ${ }^{1}$ \\ Akizuku Morikawa, ${ }^{1}$ Eiji Oshima, ${ }^{1}$ Masatomo SeKIguchI, ${ }^{1}$ \\ Kaneo IsHII, ${ }^{1}$ Isao MAKINO, ${ }^{1}$ and Kazuya MIKAMO ${ }^{2}$ \\ ${ }^{1}$ The Second Department of Internal Medicine and \\ ${ }^{2}$ Department of Biological Science, Asahikawa Medical \\ College, Asahikawa 078, Japan
}

(Received October 23, 1989)

\begin{abstract}
Summary Plasma and tissue lipid peroxide levels were examined in spontaneously diabetic Chinese hamsters (CHA colony) to elucidate the relationship between lipid peroxides and diabetes mellitus. The animals were divided into three groups: group A with severe diabetes, group B with moderate diabetes and group $\mathrm{C}$ without diabetes. The plasma lipid peroxide level of groups A and B was significantly higher than that of group $\mathrm{C}$. This level showed significant positive correlations with fasting plasma glucose and lipid levels, but a significant negative correlation with the non-fasting plasma insulin level. The lipid peroxide contents of the liver and aorta of group A were significantly higher than those of group C. We conclude that the plasma lipid peroxide level increases in these diabetic hamsters with the advance of hypoinsulinemia, hyperglycemia, and hyperlipidemia and that its content in the liver and aorta is also increased in these animals.
\end{abstract}

Key Words: lipid peroxides, diabetes mellitus, Chinese hamsters, plasma, tissue

Lipid peroxides are formed from polyunsaturated fatty acids via radicals, and have been found to be a causative factor in aging [1] and atherosclerosis [2-5]. As for the relationship between lipid peroxides and diabetes mellitus, Sato et al. [6] reported a significantly elevated plasma lipid peroxide level in diabetics, especially those with micro- or macroangiopathy (atherosclerosis). Furthermore,

*To whom correspondence should be addressed. Present address: Division of Metabolism, Endocrinology and Nutrition, RG-26, University of Washington, Seattle, WA 98195, USA. 
Sugawara et al. [7] emphasized the possible relationship between lipid peroxides and macroangiopathy in diabetes, based on their observations that although the plasma lipid peroxide level showed no difference between diabetics and healthy individuals matched for age, it was significantly elevated in diabetics over 50 years of age if they had ischemic heart disease and/or hyperlipidemia. These data suggest that elevated lipid peroxides could be one of the factors responsible for diabetic micro- and/or macroangiopathy, a disorder that occurs more frequently in diabetes than in other diseases. However, the relationship between lipid peroxides and diabetes is not fully understood.

Spontaneously diabetic Chinese hamsters (CHA colony) [8-11], which were established by Funaki and Mikamo [8], provide an animal model of non-obese, insulin-deficient diabetics. These animals are thought to be a useful model for the study of diabetic vascular diseases because of the similarity of their disease to diabetes in humans and their long diabetic lives without the need for insulin therapy.

As examination of lipid peroxide level in these spontaneously diabetic animals may be informative, in the present study we evaluated plasma and tissue lipid peroxide levels, and its associations with other parameters (glucose, insulin and lipid levels), in these animals to clarify the relationships among them.

\section{MATERIALS AND METHODS}

Chinese hamsters (CHA colony) maintained in the Department of Biological Science, Asahikawa Medical College, were employed in this study. They were kept under the following laboratory conditions: $14 \mathrm{~h}$ illumination from 5 a.m. to 7 p.m.; temperature, $23 \pm 2^{\circ} \mathrm{C}$; humidity, $50-60 \%$. They were fed normal laboratory chow (MF solid stock food, Oriental Kobo Co., Tokyo) and given water ad libitum. As shown in Table 1, they were divided into three groups according to fasting plasma glucose (FPG) levels: group A with severe diabetes (mean FPG, 449 mg/dl), group B with moderate diabetes $(184 \mathrm{mg} / \mathrm{dl})$, and group $\mathrm{C}$ without diabetes $(92 \mathrm{mg} / \mathrm{dl})$.

EDTA blood samples were collected from the orbital venous plexus after overnight fasting, and plasma was separated by centrifugation. FPG, free fatty acids (FFA), triglyceride (TG), phospholipids (PL), and total cholesterol (chol) levels were determined enzymatically $[9,11,12]$; and high-density lipoprotein (HDL)-chol levels, by the previously described microliter-scale ultracentrifugation [12]. Plasma lipid peroxide levels were determined in heparinized blood samples by the method of Yagi [13]. Non-fasting plasma insulin levels were measured by radioimmunoassay using the plain charcoal method [10].

The whole body of the animals under ether anesthesia was perfused with physiologic saline introduced from the origin of the aorta; and then the liver, thoraco-abdominal aorta, heart, kidney, and pancreas were removed. The tissue lipid peroxide contents were determined by the method of Ohkawa et al. [14].

All values were expressed as mean \pm SEM. Statistical comparisons between the 
three groups were made by analysis of variance, and those between the two groups were made by the unpaired two-tailed Student's $t$-test. A value of $p<0.05$ was accepted as significant.

\section{RESULTS}

As shown in Table 1, there was no significant difference in the age among the three groups, but the body weight was significantly $(p<0.001)$ lower in severely diabetic group A and moderately diabetic group B than in the non-diabetic control group $\mathrm{C}$. The non-fasting plasma insulin level was lowest in group $\mathrm{A}$, intermediate in group $\mathrm{B}$, and highest in group $\mathrm{C}$, with significant differences $(p<$

Table 1. Characteristics of Chinese hamsters (CHD colony).

\begin{tabular}{cccccc}
\hline Group & Number & $\begin{array}{c}\text { Age } \\
(\text { months })\end{array}$ & $\begin{array}{c}\text { Body weight } \\
(\mathrm{g})\end{array}$ & $\begin{array}{c}\text { Fasting plasma } \\
\text { glucose }(\mathrm{mg} / \mathrm{dl})\end{array}$ & $\begin{array}{c}\text { Non-fasting } \\
\text { plasma insulin } \\
(\mu \mathrm{U} / \mathrm{ml})\end{array}$ \\
\hline A & 15 & $6.8 \pm 0.6$ & $30.0 \pm 1.3^{\mathrm{a}}$ & $449 \pm 24^{\mathrm{b}}$ & $6.2 \pm 0.5^{\mathrm{b}}$ \\
$\mathrm{B}$ & 16 & $6.2 \pm 0.4$ & $30.1 \pm 1.0^{\mathrm{a}}$ & $184 \pm 8^{\mathrm{b}}$ & $50.0 \pm 8.4^{\mathrm{b}}$ \\
$\mathrm{C}$ & 15 & $6.0 \pm 0.5$ & $36.9 \pm 1.1$ & $92 \pm 3$ & $106.2 \pm 16.9$ \\
\hline
\end{tabular}

${ }^{a} p<0.001$ vs. group C, ${ }^{b} p<0.001$ vs. groups B and/or C. Mean \pm SEM. Group A, Severely diabetic group; group B, moderately diabetic group; group $\mathrm{C}$, non-diabetic control group.

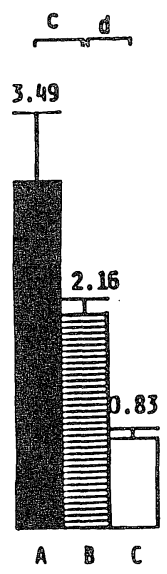
Free Facey Acld ( $\pi E Q / L)$
Triglycerlde (mg/dil
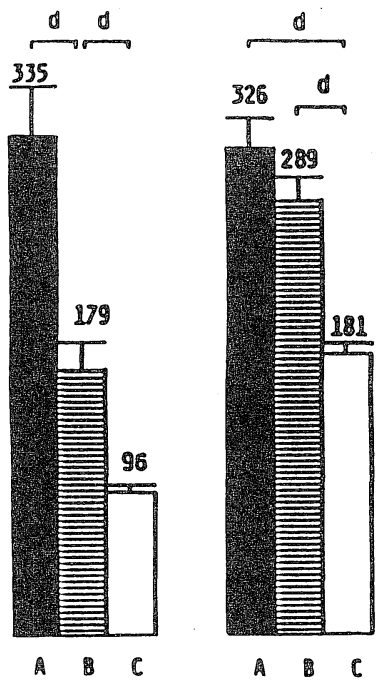

ค 6

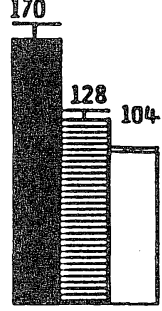

(2) C

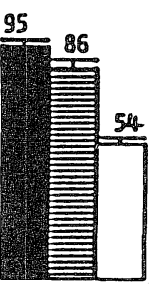

a $\mathrm{C}$

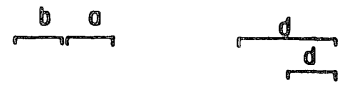

Phosohollpla (ming/d)
Total Cholesteral HDL-Cholesterol (mg/dll (img/dil)

Fig. 1. Plasma lipid levels in Chinese hamsters. Mean value $\pm S E M$ is given. Significant difference between the two groups: ${ }^{\mathrm{a}} p<0.02$; ${ }^{\mathrm{b}} p<0.01$; ${ }^{\mathrm{c}} p<0.005$; ${ }^{\mathrm{d}} p<0.001$. A, Severely diabetic group $(n=15)$; $\mathrm{B}$, moderately diabetic group $(n=16)$; $\mathrm{C}$, non-diabetic control group $(n=15)$. 


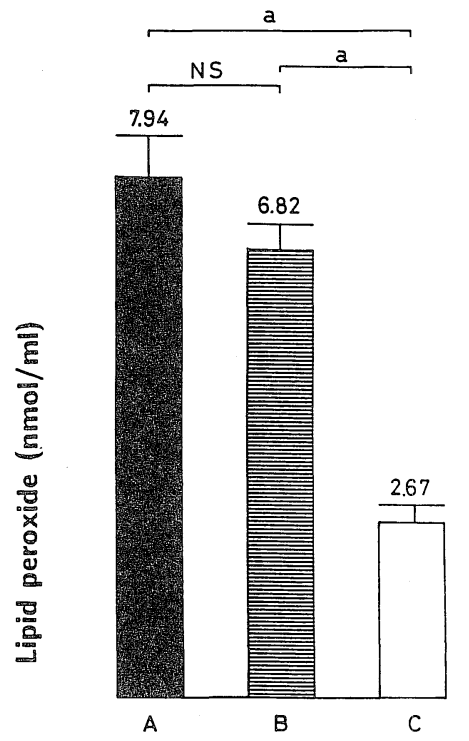

Fig. 2. Plasma lipid peroxide level in Chinese hamsters. Mean value $\pm S E M$ is given. Significant difference between the two groups: ${ }^{a} p<0.001$. NS, Not significant. A; Severely diabetic group $(n=15)$; $\mathrm{B}$, moderately diabetic group $(n=16)$; , non-diabetic control group $(n=15)$.

Table 2. Correlation between plasma lipid peroxide level and various parameters.

\begin{tabular}{lc}
\hline Parameter & $r$ \\
\hline FPG & $0.638^{\mathrm{a}}$ \\
Insulin* & $-0.612^{\mathrm{a}}$ \\
Free fatty acids & $0.447^{\mathrm{a}}$ \\
Triglyceride & $0.587^{\mathrm{a}}$ \\
Phospholipids & $0.771^{\mathrm{a}}$ \\
Total cholesterol & $0.678^{\mathrm{a}}$ \\
HDL-cholesterol & $0.769^{\mathrm{a}}$ \\
\hline a $p<0.01$ FPG, Fasting plasma glucose *Non-fasting plasma insulin.
\end{tabular}

0.001) among the three groups.

The plasma FFA, TG, PL, total chol, and HDL-chol levels were significantly higher in groups A and B than in group C (Fig. 1), and the FFA, TG, and total chol levels were significantly higher in group A than in group B. The values of group B always tended to be intermediate between those of groups $\mathrm{A}$ and $\mathrm{C}$.

As shown in Fig. 2, the plasma lipid peroxide level of groups $\mathrm{A}$ and $\mathrm{B}$ was significantly $(p<0.001)$ higher than that of group $\mathrm{C}$, but the difference between groups $\mathrm{A}$ and $\mathrm{B}$ was not significant.

The plasma lipid peroxide level showed significant $(p<0.01)$ positive correlations with FPG, plasma FFA, TG, PL, total chol, and HDL-chol levels, but a significant $(p<0.01)$ negative correlation with the non-fasting plasma insulin 


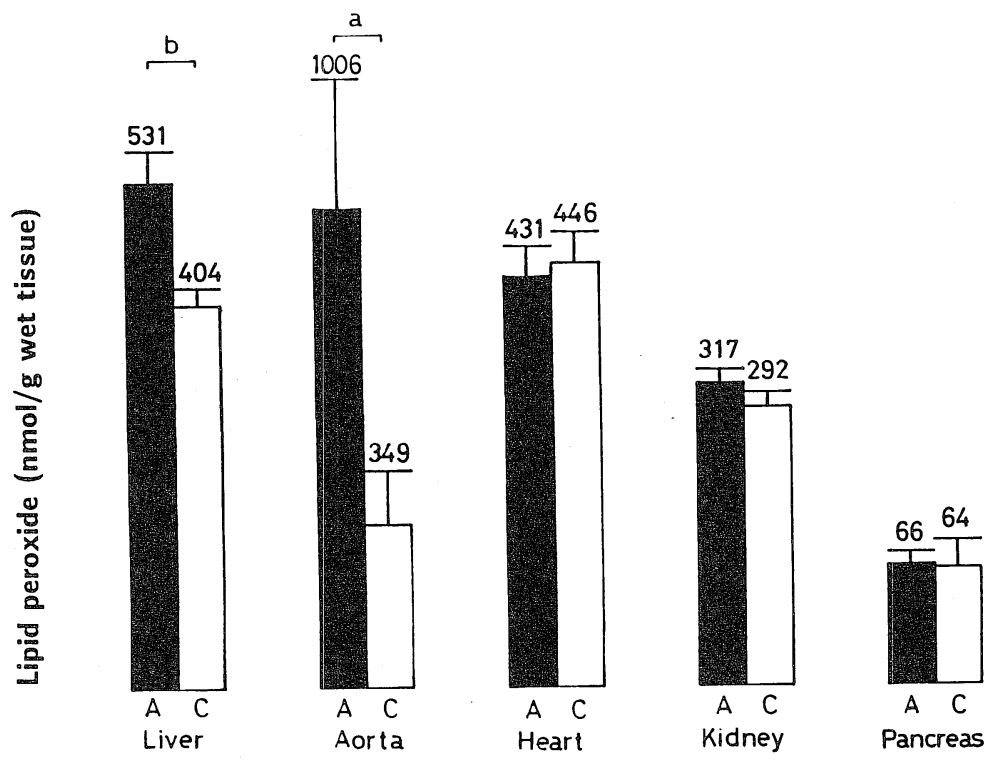

Fig. 3. Tissue lipid peroxide content in Chinese hamsters. Mean value \pm SEM is given. Significant difference between the two groups: ${ }^{\mathrm{a}} p<0.05$; ${ }^{\mathrm{b}} p<0.01$. A, Severely diabetic group $(n=6)$; C, non-diabetic control group $(n=6)$.

level (Table 2).

The tissue lipid peroxide contents were determined only in groups $\mathrm{A}$ and $\mathrm{C}$, and thus comparison was made between these two groups only. The lipid peroxide contents of the liver and aorta of group A were significantly $(p<0.01$ and $p<0.05$, respectively) higher than those of group C (Fig. 3). However, the lipid peroxide contents of the heart, kidney, and pancreas showed no difference between the two groups.

\section{DISCUSSION}

The plasma lipid peroxide level of these diabetic hamsters was significantly higher than that of the non-diabetic animals. Its elevated level has also reported in diabetic humans [6], but this observation has not yet attained general acceptance [7], probably due to effects of such multifarious factors in diabetic humans as age, diet, therapy mode, alcohol consumption, and smoking habit. Because our diabetic animals are homogenous, the present study strongly supports the elevation of the plasma lipid peroxide level in diabetes.

In this study, we also prove that the plasma lipid peroxide level increased with the advance of hypoinsulinemia and hyperglycemia. These animals develop diabetes due to the disruption or dysfunction of $\beta$-cells in the islets [10], which results in hypoinsulinemia. Although the cause of the elevated lipid peroxide level 
in our diabetic animals remains obscure, hypoinsulinemia followed by hyperglycemia may be primarily related to this elevation. The plasma lipid peroxide level also increased with the advance of hyperlipidemia [12]. There is a possibility that the increased lipid peroxide level can be attributed to the hyperlipidemia. Earlier we reported hyperlipidemia in diabetic hamsters in details [12]. The increased HDL-chol level seen in diabetic hamsters seems not to occur in human diabetes. Plasma low-density lipoprotein (LDL) scarcely exists in hamsters [12].

The lipid peroxide content was increased in the liver of the diabetic animals. It is possible that insulin deficiency may cause metabolic disordes that induce an increase in the synthesis of lipid peroxides by the liver, its release into circulation, and a resultant elevation of its circulating concentration. The liver seems important as the primary source of lipid peroxides in the diabetic state.

An increased lipid peroxide content was also noted in the aorta of the diabetic hamsters. We already showed earlier that the aortic intima of these diabetic animals has morphological abnormalities such as cell proliferation, slight thickening, and swelling, which may be an early phase of atherosclerosis [15]. These observations, in addition to the elevated plasma lipid peroxide level, suggest the possible relationship between lipid peroxides and atherosclerosis in diabetes, as reported previously in diabetic humans $[6,7]$. Whether its increased content in the aorta was due to the transport from blood with increased lipid peroxides or to the synthesis in the aorta or both is not clear. As for the relationship between lipid peroxides and atherosclerosis, Glavind et al. [2] detected lipid peroxides in human atherosclerotic lesions, and Cutler and Schneider [16] observed an increase in lipid peroxide content and appearance of plaques in the rabbit aortic wall following repeated subcutaneous administration of linoleate hydroperoxide. Furthermore, Yagi $[4,5]$ reported that increased serum lipid peroxides initiate atherogenesis. Lipid peroxides may be also atherogenic because they induce degeneration of LDL [17]. Further studies are needed to clarify whether lipid peroxides are involved in the pathogenesis of atherosclerosis in diabetes.

We previously found thickening of the renal glomerular basement membrane, (i.e., diabetic nephropathy) in diabetic hamsters $[9,18]$. However, our present study revealed no difference in the renal lipid peroxide content. Much remains to be clarified concerning the relationship between lipid peroxides and diabetic microangiopathy. The pancreatic lipid peroxide content also showed no difference despite a report of adverse effects of lipid peroxides on pancreatic Langerhans islets [19]. Finally, we reported the presence of cardiomyopathy in the diabetic hamsters [11], however, this disorder is probably not related to lipid peroxides because we detected no change in the cardiac content of the peroxide in these animals.

This study was supported in part by a Grant-in-Aid for scientific research (No. 57771586) from the Ministry of Education, Science and Culture of Japan. 


\section{REFERENCES}

1. Hagihara, M., Nishigaki, I., Maseki, M., and Yagi, K. (1984): Age-dependent changes in lipid peroxide levels in the lipoprotein fractions of human serum. J. Gerontol., 39, 269-272.

2. Glavind, J., Hartmen, S., Clemmesen, J., Jessen, K.E., and Dam, H. (1952): Studies on the role of lipoperoxides in human pathology. Acta Pathol. Microbiol. Scand., 30, 1-6.

3. Yagi, K. (1982): Assay for serum lipid peroxide level and its clinical significance, in Lipid Peroxides in Biology and Medicine, ed. by Yagi, K., Academic Press, New York, pp. 223242.

4. Yagi, K. (1984): Increased serum lipid peroxides initiate atherogenesis. BioEssays, 1, 58-60.

5. Yagi, K. (1986): A biochemical approach to atherogenesis. Trends Biochem. Sci., 11, 18-19.

6. Sato, Y., Hotta, N., Sakamoto, N., Matsuoka, S., Ohishi, N., and Yagi, K. (1970): Lipid peroxide level in plasma of diabetic patients. Biochem. Med., 21, 104-107.

7. Sugawara, K., Yoda, T., Hirata, T., and Goto, Y. (1981): Diabetes mellitus and plasma lipid peroxides. J. Jpn. Diab. Soc., 24, 891-896.

8. Funaki, K., and Mikamo, K. (1983): Development stage-dependent teratogenic effects of maternal spontaneous diabetes in the Chinese hamster. Diabetes, 32, 637-643.

9. Watanabe, K., Morikawa, A., Eto, M., Iwashima, Y., Takebe, T., and Ishii, K. (1984): Characterization of diabetic Chinese hamsters in the Asahikawa colony, in Lessons from Animal Diabetes, ed. by Shafrir, E., and Renold, A.E., John Libbey, London and Paris, pp. 99- 105.

10. Iwashima, Y., Watanabe, K., Eto, M., Morikawa, A., Takebe, T., and Ishii, K. (1985): Insulin and glucagon response of the diabetic Chinese hamster in the Asahikawa colony. Diabetes Res. Clin. Pract., 1, 87-94.

11. Eto, M., Watanabe, K., Sekiguchi, M., Iwashima, Y., Morikawa, A., Oshima, E., and Ishii, K. (1987): Metabolic and morphological changes of the heart in Chinese hamsters (CHAD strain) with spontaneous long-term diabetes. Diabetes Res. Clin. Pract., 3, 297-305.

12. Eto, M., Watanabe, K., Iwashima, Y., Morikawa, A., Takebe, T., and Ishii, K. (1984): Elevation of plasma high density lipoprotein-cholesterol in spontaneously diabetic Chinese hamsters. Tohoku J. Exp. Med., 144, 281-290.

13. Yagi, K. (1976): A simple fluorometric assay for lipoperoxide in blood plasma. Biochem. Med., 15, 212-216.

14. Ohkawa, H., Ohishi, N., and Yagi, K. (1979): Assay for lipid peroxides in animal tissues by thiobarbituric acid reaction. Anal. Biochem., 95, 351-358.

15. Eto, M., Watanabe, K., Iwashima, Y., Morikawa, A., Takebe, T., and Ishii, K. (1984): Plasma lipids, lipoproteins and apoproteins in spontaneously diabetic Chinese hamsters. $J$. Jpn. Atheroscler. Soc., 12, 281-289.

16. Cutler, M.G., and Schneider, R.: Linoleate oxidation products and cardiovascular lesions. Atherosclerosis, 20, 383-394.

17. Nishida, T., and Kummerow, F.A. (1960): Interaction of serum lipoproteins with hyperoxide of methyl linoleate. J. Lipid Res., 1, 450-458.

18. Morikawa, A., Watanabe, K., Eto, M., Iwashima, Y., Takebe, T., and Ishii, K. (1983): Renal glomerular lesions in spontaneously diabetic Chinese hamsters (electron microścopic analysis). J. Jpn. Diab. Soc., 26, 436.

19. Nagano, S., Nishimura, T., Fukuyama, S., and Hirano, T. (1983): Cytotoxicity of pancreas Langerhans islets by lipid peroxides. Igakunoayumi, 127, 728-730. 